\title{
Gewappnet für die Zukunft
}

\author{
Ute Buschmann ${ }^{a}$, \\ Thomas Kaufmann ${ }^{b}$, \\ Herbert Lienhardc, \\ David Baumann, \\ Joachim Diebolde, \\ Guido Schüpferf
}

a Fachärztin für Neurochirurgie, NDS Qualitätsmanagement HF, Riskmanagerin Luzerner Kantonsspital

b NDS für Betriebswissenschaften BWI, ETH Zürich, Leiter Abteilung Qualitätsund Riskmanagement Luzerner Kantonsspital

c Geschäftsführer aaarisk GmbH, Güttingen

d Qualitätsmanager NDS Qualitätsmanagement $\mathrm{HF}$ Pathologisches Institut, Luzerner Kantonsspital

e Chefarzt Pathologisches Institut, Luzerner Kantonsspital

f Executive MBA HSG, Co-Chefarzt Anästhesie, Leiter Stab Medizin Luzerner Kantonsspital

1 Barodte B, Montagne E, Fischer A. Eingeschränkter Blick. Harvard Business manager. Nov 2007; S.8.

2 Gleissner W, Lienhard $\mathrm{H}$, Stroeder D. Risikomanagement im Mittelstand Planungssicherheit erhöhen, Rating verbessern, Unternehmen sichern - RKW e.V. 2004; S.9-24.

Korrespondenz:

Dr. med. Ute Buschmann Luzerner Kantonsspital

Abteilung Qualitäts- und

Riskmanagement

CH-6000 Luzern 16

Tel. 0412052511

ute.buschmann@ksl.ch
Mit zirka 11\% des Bruttoinlandprodukts (BIP) ist das Gesundheitswesen einer der wichtigen Sektoren der Schweizerischen Volkswirtschaft, der knapp zur Hälfte von der stationären Versorgung dominiert wird. Spitäler bewegen sich in einem komplexen und stark regulierten Markt. Eine rechtliche Verselbständigung oder «Privatisierung» der Spitäler führt dazu, dass sie zusätzlich gesellschaftsrechtlichen Regelungen und zunehmend Marktprinzipien unterliegen. Das Risikomanagement wird als ergänzendes Führungsinstrument zum unverzichtbaren und elementaren Baustein einer zukunftsorientierten und umfassenden Unternehmenssteuerung. Der Stellenwert ist mindestens mit dem der Finanzen vergleichbar. Durch proaktives, zukunftsgerichtetes Risikomanagement sollen die Planungssicherheit erhöht und letztlich Wettbewerbsvorteile generiert werden. Für den Erfolg eines Unternehmens ist es wesentlich, sowohl interne als auch externe Risiken in den Strategieplanungsprozess einfliessen zu lassen [1]. Durch Offenlegung auch von Chancen durch das Risikomanagement wird die $\mathrm{Zu}$ kunftsfähigkeit eines Unternehmens entscheidend verbessert [2, 3].

\section{Gesetzlicher Hintergrund - \\ Art. 663b Ziff.12 OR}

Im neuen Obligationenrecht wurden die Bestimmungen zum internen Kontrollsystem (IKS) sowie zur Risikobeurteilung neu geregelt. Gemäss Art. 663b Ziff.12 OR (Obligationenrecht) wird seit 2008 von Unternehmen, die einer ordentlichen bzw. einer eingeschränkten Revision unterliegen, verlangt, im Anhang der Jahres- und Konzernrechnung Angaben über die Durch-

\section{Unternehmensprofil des Luzerner Kantonsspitals}

Als Zentrumsspital der Zentralschweiz, bestehend aus den Standorten Luzern, Wolhusen, Sursee sowie der Klinik Montana, stellt das Luzerner Kantonsspital (LUKS) die medizinische Versorgung für mehr als 500000 Personen aus dem direkten Einzugsgebiet sicher. Die Anzahl der Patientenaustritte liegt pro Jahr bei über 36000 , mit steigender Tendenz. Es bestehen zahlreiche Kooperationen mit weiteren Spitälern. Das LUKS ist mit mehr als 3400 Vollzeitstellen (ohne Ausbildungsstellen) der grösste Arbeitgeber der Zentralschweiz.

\section{Préparé pour l'avenir}

Jusqu'à aujourd'hui, beaucoup d'entreprises ont fondé leur politique de gestion en s'appuyant sur les informations et les exercices des années précédentes.

Aussi, les décisions ont souvent été prises en retard et les développements techniques et de mise sur le marché ont été insuffisamment anticipés.

Avant tout, un changement de paradigme apporte de nouveaux bénéfices à l'entreprise. Néanmoins, les risques encourus doivent être connus et la pérennité de l'entreprise est garantie.

Une projection dans le futur permet d'apprécier les chances. Les moyens expérimentés proposés par le Riskmanagement permettent d'identifier, d'analyser et d'évaluer les risques et les chances de succès. Le Riskmanagement offre des critères de choix indispensables au développement de stratégies nouvelles, durables et ciblées.

führung einer Risikobeurteilung zu machen. Diese liegt im Verantwortungsbereich des Verwaltungsrates. Bezüglich Umfang und Detaillierungsgrad der Angaben wird die Art, Grösse, Komplexität und Risikosituation des Unternehmens als bestimmend angesehen [4].

\section{Anforderungen und Nutzen}

Um für die erwarteten Veränderungen im Gesundheitswesen gewappnet zu sein, zu denen insbesondere auch die Einführung des Swiss-DRG-Systems zählt, entschieden Spitalrat und Geschäftsleitung des LUKS im Jahr 2009, das Risikomanagement als integralen Bestandteil der Unternehmenssteuerung einzuführen. Das angestrebte Risikomanagementsystem soll dabei deutlich über die gesetzlichen Bestimmungen hinausgehen, um eine nachhaltige Wirkung entfalten zu können. Neben formalen Aspekten wurde als wesentliche Anforderung auch der Aufbau einer breit abgestützten Risikokultur formuliert. 
3 Kaub M, Schäfer M. Wertorientierte Unternehmensführung. Die Berichterstattung der Abschlussprüfer. Fachhochschule für Wirtschaft in Berlin i. A. der Hans-Böckler-Stiftung. 2002

4 Pfaff D. IKS-Leitfaden Empfehlungen des veb.ch zum internen Kontrollsystem IKS und zu den Angaben über die Risikobeurteilung im Anhang. 2008; S. 7-9, S.18-20.
Die formalen und ökonomischen Anforderungen an ein integrales Risikomanagement sind - Gesetzeskonformität (Erfüllung Art. 663b Ziff.12 OR)

- Fokussierung auf die relevantesten Risiken aller Bereiche

- Keine zusätzliche Vorbereitung der Risikoverantwortlichen

- Quantifizierbarkeit der Risiken und Gesamtrisikoberechnung

- Frühzeitige Erkennung bestandesgefährdender Entwicklungen im Unternehmen

- Integration bestehender Controlling- und Planungsprozesse (z. B. IKS, IT-Sicherheit)

- Klar geregelte Verantwortlichkeiten

- Aufbau eines Risikobewusstseins im ganzen Unternehmen

- Unterstützung der Unternehmenssteuerung zur Verbesserung der Wertschöpfung

\section{Abbildung 1}

Risikomanagementorganisation: Das Risikomanagement koordiniert und unterstützt die Risikoverantwortlichen, die eigentlichen Experten, administrativ und methodisch und garantiert eine konsistente Beurteilung der Risiken.

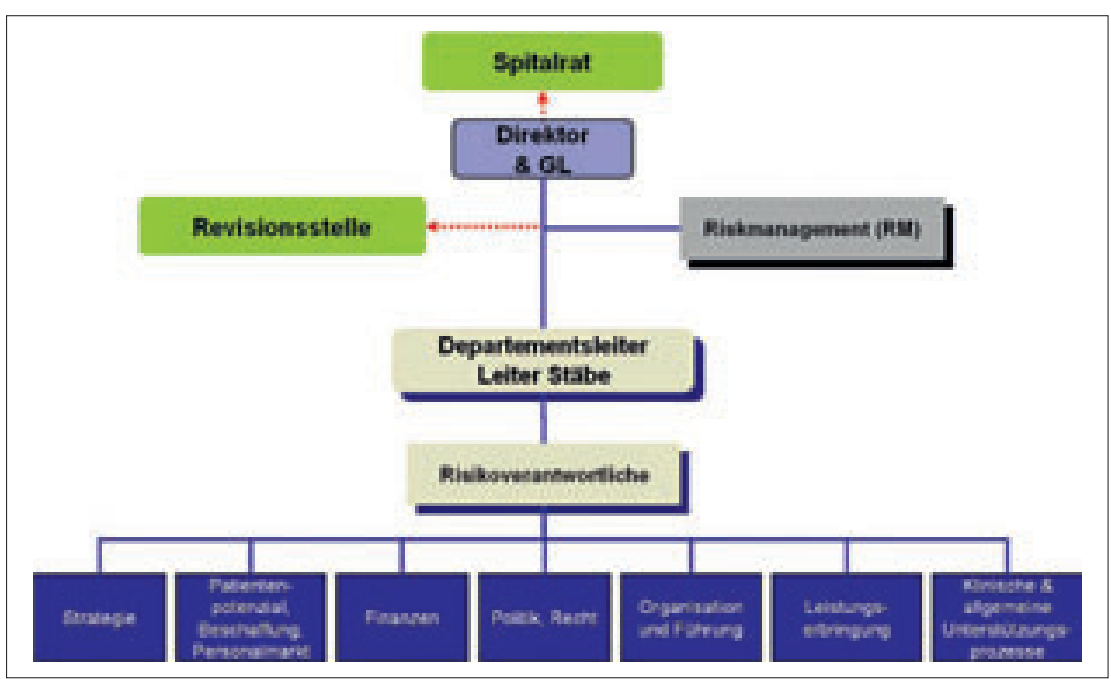

Abbildung 2

Risikomanagementprozess: Das periodische Durchlaufen des Prozesses gewährleistet ein systematisches Controlling und eine kontinuierliche Optimierung der Risikobewältigung

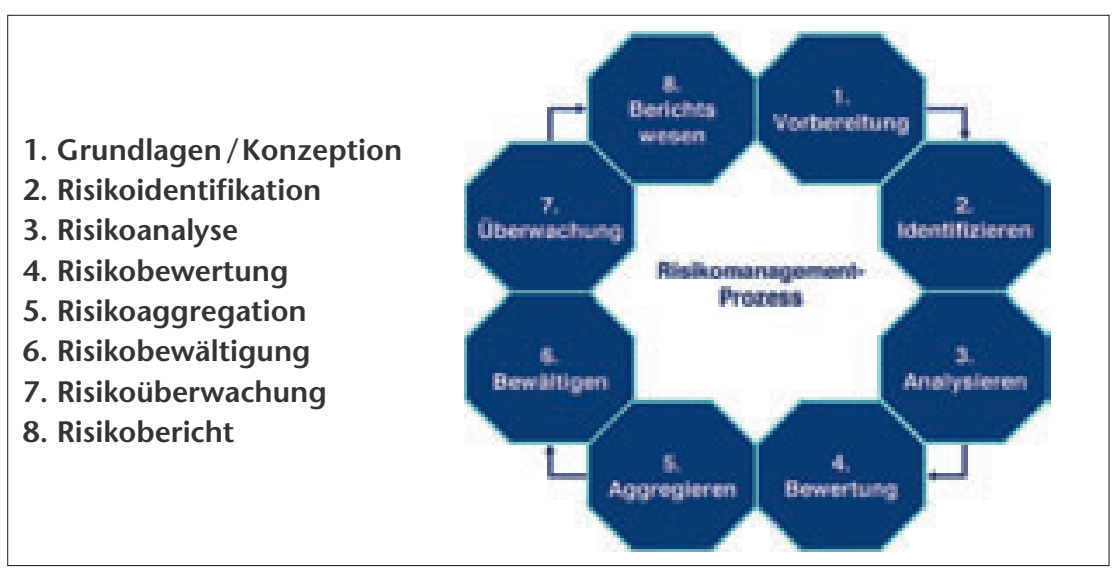

\section{Organisation und Verantwortlichkeiten}

Die Projektleitung zur Einführung des Risikomanagements wurde der Abteilung Qualitäts- und Risikomanagement des LUKS übertragen, die im Stab Medizin verankert ist. Das Risikomanagement ist ebenfalls für die Patientensicherheit, das spitalweite CIRS und das Haftpflichtmanagement verantwortlich, wodurch die Kompetenz konzentriert wird. Zum Kompetenzaufbau wurde das Projektteam durch einen externen Berater unterstützt.

Während die Verantwortung für die erfolgreiche Implementierung und Etablierung des integralen Risikomanagement-Systems beim Qualitäts- und Risikomanagement liegt, sind die Risikoverantwortlichen zuständig für die ihnen zugeteilten sogenannten Risikofelder sowie die identifizierten Risiken inklusive deren Bewältigung. Bei den Risikoverantwortlichen handelt es sich um Kaderpersonen der verschiedenen Unternehmensbereiche wie z. B. Chefärzte, Abteilungsleiter, Pflegekader usw. Geschäftsleitung und Spitalrat tragen die Gesamtverantwortung für den Umgang mit den ermittelten Risiken am LUKS (s. Abb. 1).

\section{Der Risikomanagementprozess}

Der Risikomanagementprozess gliedert sich im Wesentlichen in 8 Schritte, die im Sinne eines kontinuierlichen Verbesserungsprozesses jährlich durchlaufen werden (s. Abb. 2).

Als wesentliche Voraussetzung zur Erarbeitung des Gesamtprozesses dienen die initial erhobenen Grundlageninformationen im Unternehmen. Dazu zählen insbesondere die Gesamtunternehmensstrategie, Budget- und Planzahlen, wirtschaftliche Eckdaten der letzten drei Jahre, Ablaufpläne der wesentlichen Unternehmensprozesse sowie die Organisationsstrukturen. Auf der Basis dieser Grundinformationen wurden der Zeit- und Ressourcenplan und auch die Projektorganisation festgelegt. Des Weiteren werden die Risikofelder definiert und die unternehmensspezifische fünfstufige Relevanzskala abgeglichen. Die Relevanzskala orientiert sich am längerfristig notwendigen Betriebsergebnis und dient der Priorisierung der Einzelrisiken. Durch diese Schwellenwerte wird auch sichergestellt, dass sich das Risikomanagement auf die wesentlichen Risiken konzentriert (s. Abb. 3).

Das Kernstück des Risikomanagementprozesses stellt die Erhebung des Risikoprofils dar (s. Abb. 2 Schritt 2-5). Entsprechend den Kategorien Strategie/Patientenpotential, Beschaffung, Personalmarkt/ Finanzen/Politik und Recht/Organisation und Führung/Leistungserbringung/Klinische Unterstützungsprozesse/Allgemeine Unterstützungsprozesse werden differenzierte Risikofelder ausgearbeitet. Bei der Leistungserbringung und den klinischen Unterstützungsprozessen werden alle medizinischen Fachbereiche berücksichtigt. Die Erhebung der Risiken inklusive vorhandener bzw. möglicher Bewältigungsmassnahmen sowie die Bewertung und Priorisierung der Risiken 
anhand der Relevanzskala erfolgt gemeinsam mit den jeweiligen Risikoverantwortlichen im teilstrukturierten Interviewverfahren. Die Risikoidentifikation wird durch Checklisten unterstützt.

\section{Abbildung 3}

Relevanzskala zur Priorisierung der Einzel- und Gesamtrisiken. Die Abstufung ist abhängig vom Betriebsergebnis des Gesamtunternehmens und wird für alle Bereiche konsistent angewendet.

\begin{tabular}{|c|c|}
\hline \multicolumn{2}{|r|}{ Rolevanzskala } \\
\hline Basiegrosea & definiertes Betricbsergebnis \\
\hline Pelevanz & Ausprasung \\
\hline 1 & 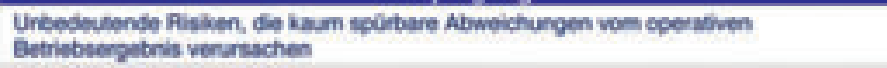 \\
\hline 2 & Mittere Risken, de spötare Abweichungen vom cperwhen Betriabsergobnis bewiken \\
\hline 3 & $\begin{array}{l}\text { Bedeutende Risien, de das operativen Betriebsergebis stark beeirhusen eder sich } \\
\text { auch langfristig ausairken }\end{array}$ \\
\hline 4 & $\begin{array}{l}\text { Schwerwiegende Aisken, die zu grossen Abweichungen von operativen } \\
\text { Betriebsergetris when ind / oder sich auch larghtistig erhebilch aumwirken }\end{array}$ \\
\hline 5 & 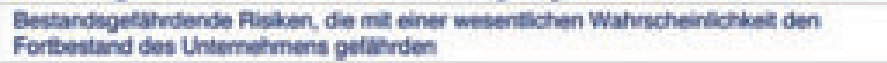 \\
\hline
\end{tabular}

\section{Abbildung 4}

Verringerung der Gewinnstreuung: Durch Optimierung der Risikobewältigungsmassnahmen verringert sich die Gewinnstreuung und somit die Verlustwahrscheinlichkeit sowie der Eigenkapitalbedarf zur Risikodeckung.

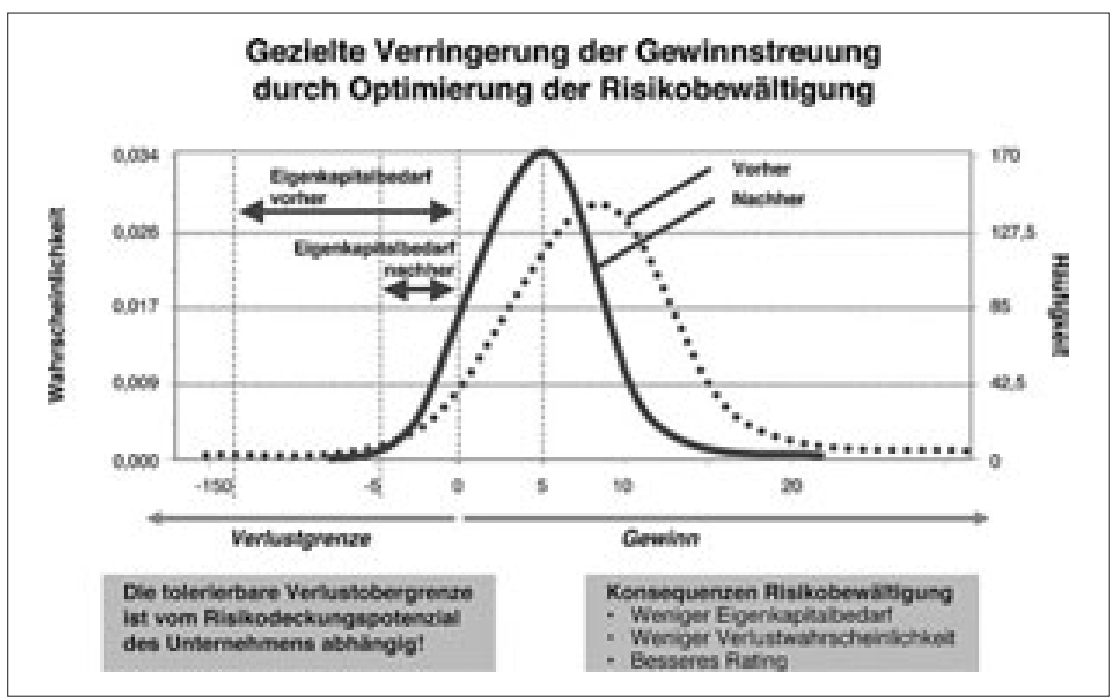

\footnotetext{
Diskussionspunkte bei der Risikoanalyse im Pathologischen Institut

- Bedarfsermittlung, Abstimmung,

Koordination

- Apparaturen, Instrumentarium, Infrastruktur

- Kompetenzprofil, Know-how

- Leistungsprofil, Marktsituation

- Personalmanagement

- Kostenmanagement

- Leistungserstellung

- Qualitäts- und Prozessmanagement

- Rechtliche Belange, Haftung $\longrightarrow$

- CIRS

- Notfallmanagement
}

Zur weiteren Bearbeitung und Bewertung werden alle Risiken mittels der speziellen Software «RisikoKompass» erfasst und zu einem Gesamtinventar konsolidiert.

Mittels Risikoaggregation wird die Gesamtrisikoposition des LUKS berechnet. Dabei werden die kalkulatorisch jährlich wiederkehrenden Risikokosten sowie das Gesamtrisiko in Form eines Höchstschadenswertes ausgewiesen, der zu einer bestimmten Wahrscheinlichkeit, z.B. $95 \%$, nicht überschritten wird. Durch die Angabe des Höchstschadenswertes lassen sich die Risikotragfähigkeit und der Risikofinanzierungsbedarf des Unternehmens ermitteln und jährlich vergleichen.

Durch die konsequente Umsetzung von Risikobewältigungsmassnahmen wird die Planungssicherheit wesentlich erhöht, der Risikofinanzierungsbedarf gesenkt und die Risikotragfähigkeit verbessert (s. Abb. 4). Das ermittelte Risikoinventar sowie die Gesamtrisikoabschätzung und das Risikokennzahlen-Tableau ergänzen und beeinflussen die Unternehmenssteuerung sinnvoll. Das Gesamtrisikoinventar sowie die bewerteten und aggregierten Risiken fliessen in den jährlichen internen Risikobericht ein.

\section{Umsetzung in der Praxis am Beispiel des Pathologischen Instituts}

Das Pathologische Institut am LUKS bietet Spitzenqualität auf Universitätsniveau. Entsprechend hoch ist auch der externe Kundenanteil.

Bei der übergeordneten Risikoanalyse wurden im Interview mit dem Chefarzt und dem Qualitätsmanager des Pathologischen Instituts sowohl interne als auch externe Risiken beleuchtet (Kriterien s. Kasten). Dabei konnte auf einer im Rahmen der Akkreditierung bereits durchgeführten detaillierten Bereichsanalyse der Strategie-, Management- und Prozessrisiken aufgebaut werden. Nachfolgend wird der Kernprozess «Histologie» genauer betrachtet.

Aufgrund des Risikos, durch eine fehlerhafte Probenverarbeitung die korrekte Diagnose und somit die korrekte Weiterbehandlung eines Patienten zu gefährden, wurde dieses Prozessrisiko auch in der übergeordneten Risikoanalyse als kritisch eingestuft (s. Relevanzskala Abb. 3). Zu berücksichtigen ist dabei insbesondere eine sich möglicherweise langfristig sowohl auf das Pathologische Institut als auch auf verschiedene, abhängige Kliniken des LUKS auswirkende Imageeinbusse. Darüber hinaus wäre auch mit einem Rückgang des externen Kundenanteils zu rechnen.

\section{Kritische Schnittstellen im Histologieprozess}

Die Störanfälligkeit des Prozesses mit einer reellen Verwechslungsgefahr ergibt sich aus den kritischen manuellen Schnittstellen bei einer grossen Anzahl Proben täglich, die teilweise zudem als Sammellieferungen eintreffen und im Prozess manuell nummeriert werden müssen (s. Abb. 5). Dabei erschweren die zwangsläufig auftretenden Ähnlichkeiten der Nummerierung die Sicherheitskontrollen. 


\section{Prozessoptimierung -}

\section{durchgängiges Barcodesystem}

Die Detailanalyse zeigte, dass die vorhandenen Möglichkeiten der Prozessanpassungen inklusive Kontrollmechanismen zur Optimierung des Histologieprozesses bereits ausgeschöpft waren. Um eine Verbesserung der Prozesssicherheit zu erzielen, wurde nach neuen Möglichkeiten insbesondere zur Vereinfachung des Gesamtprozesses sowie zur Reduktion der Schnittstel- durch die erstmalige Risikoerhebung das Risikobewusstsein innerhalb des LUKS bei allen Beteiligten geschärft werden. Das Vorgehen anhand von Risikofeldern führte zur Definition von klaren Risikoverantwortlichkeiten und trägt wesentlich zum Aufbau einer unternehmensweiten Risikokultur bei. Der Verzicht auf eine vorgängige Vorbereitung der Interviews durch die Risikoverantwortlichen wirkte sich keinesfalls nachteilig aus. Vielmehr zeigte sich deutlich,

\section{Mit dem Blick auf die Chancen wird das Risikomanagement der Zukunft für die Spitäler einen eindeutigen Mehrwert erzielen und Wettbewerbsvorteile generieren}

len-bedingten Fehleranfälligkeit gesucht und infolgedessen die Möglichkeit eines durchgängigen Barcodesystems evaluiert. Neben der Effizienzsteigerung im Gesamtprozess werden der Wegfall der manuellen Beschriftung und Nummerierung sowie die scannerunterstützte Probenkontrolle an jeder Schnittstelle als wesentliche Sicherheitsbausteine zur Reduktion der Verwechslungsgefahr angesehen. Die Implementierung des Barcodesystems befindet sich derzeit in der Vorbereitungsphase.

Das Beispiel der Pathologie zeigt, dass durch die übergeordnete Risikopriorisierung für das Gesamtunternehmen relevante Risiken in den einzelnen Kliniken und Bereichen identifiziert und mit gezielten Massnahmen reduziert werden können.

\section{Fazit - Erfolgsfaktoren und wesentliche Erkenntnisse aus der Sicht eines Spitals}

Durch den kombinierten Einbezug der Geschäftsleitungsebene sowie aller Organisationseinheiten gemäss Organigramm wurden sowohl interne als auch externe Risiken «top-down» und «bottom-up» erfasst und so ein möglichst lückenloses Risikoinventar erstellt. Zudem konnte durch die breite thematische Durchflechtung auf verschiedenen Funktionsstufen bereits

\section{Abbildung 5}

Kritische Schnittstellen im Histologieprozess.

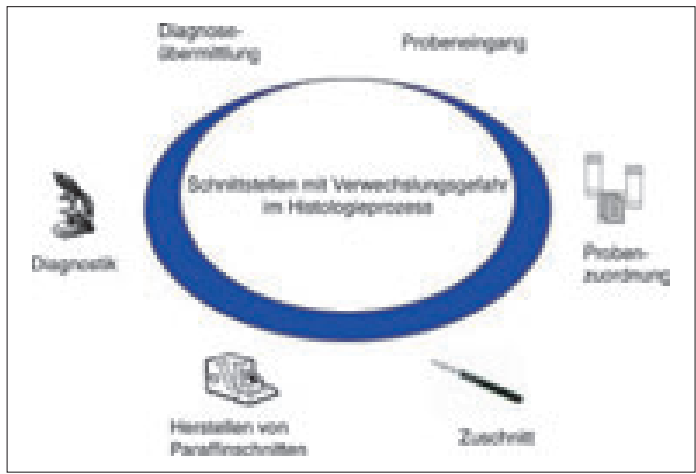

dass die Risikoverantwortlichen sehr wohl in der Lage sind, die Risiken ihres Kompetenzbereichs zu identifizieren und zu bewerten. Die Übernahme der administrativen Vor- und Nachbereitung der Interviews durch das Risikomanagement wurde von den Verantwortlichen ausserordentlich geschätzt und führte zu einer deutlichen Ressourcenschonung insbesondere bei den beteiligten Klinikern.

Die Etablierung eines integralen Risikomanagements unterliegt einem kontinuierlichen Prozess. Die zukünftige Herausforderung der Geschäftsleitung besteht darin, den Risikomanagementprozess unter Umsetzung der definierten Bewältigungsmassnahmen konsequent zu durchlaufen und die gewonnene Transparenz nachhaltig für den Strategieprozess und somit für die Unternehmenssteuerung zu nutzen und somit die Wertschöpfung des Unternehmens langfristig zu optimieren.

\section{Lessons learned}

- Die Interviews sollten mit den direkten Linienverantwortlichen geführt werden. Diese kennen die Risiken ihres Bereichs am besten.

- Die Bewertung der Risiken muss konsistent und praxisgerecht sein.

- Im Spital liegen heutzutage die grössten Risiken zum einen im Bereich der Strategie, konkret im Leistungsangebot, zum anderen im Bereich der IT. Letzteres ist durch die Komplexitätszunahme bedingt und erfordert - analog der Rechnungsrevision - eine kontinuierliche unabhängige Bewertung.

- Durch die Risikopriorisierung mit Fokus auf das Gesamtunternehmen können Ressourcen zur Risikobewältigung gezielt eingesetzt werden.

- Die Suche nach Risiken lässt unmittelbar auch Chancen erkennen. Dadurch wird die proaktive Unternehmensführung unterstützt. 